\title{
Das elektronische Diskussionsforum in der Sprachvermittlung
}

\author{
ISABELLA LEIBRANDT \\ Instituto de Idiomas \\ Universidad de Navarra
}

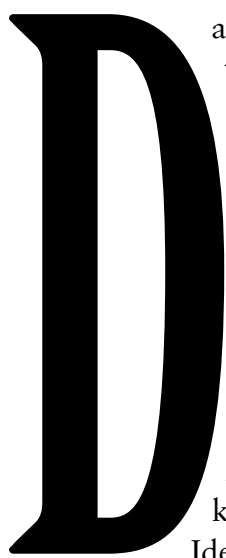

as Diskussionsforum, ein elektronisches Hilfswerkzeug, wird von seinen Teilnehmern als Weg zur Kommunikation benutzt, ist somit Ort des Austausches an Meinungen und Beiträgen zu einem gegebenen Thema im virtuellen Raum. Da es sich um ein asynchronisches Werkzeug handelt, unabhängig von Zeit und Ort, können Kommentare, Fragen, Ideen spontan und vom eigenen Computer aus von allen Winkeln auf der Welt geschickt werden. Seine Attraktivität liegt darin, dass ein virtueller Ort die Teilnehmer über die Grenzen eines Klassenraumes, einer Stadt, eines Landes verbindet und zum sozialen Treffpunkt für den Austausch an Informationen wird.

Im Folgenden sollen Aspekte seiner Nutzung vorgestellt werden, die den Deutschunterricht auf bereichernde Weise ergänzen wollen.

\section{Interkultureller Aspekt}

Von der ersten Stunde an kann das Forum für den ersten Kontakt mit den deutschsprachigen Ländern genutzt werden. Das Motiv ist ein globaler kultureller Bildungsaspekt mit dem Ziel, die Deutschlerner mit den Ländern, deren Sprache sie gerade beginnen zu lernen, in Kontakt zu setzen. Die Lerner, auch wenn zunächst noch auf Spanisch, sollen sich auf kurze virtuelle Reisen begeben und neue Welten entdecken. Als Einstieg in Kultur, Geschichte, Land und Leute kann die Webseite http://www.tatsachen-ueber-deutschland.de/es/home1.html besucht werden, auf der sich Deutschland auf zahlreichen Gebieten präsentiert. Die Lerner bekommen den Auftrag sich ein Thema auszusuchen und im Forum zu kommentieren, was sie entdeckt haben. Internet bietet sich als virtueller Ersatz für direkte Erfahrungen mit dem Land an und das Forum, um erste Informationen darüber auszutauschen.

Zwei Beispiele aus dem Forum mögen das Ergebnis dieser kleinen Forschungsreisen widergeben. So schreibt Idoia:

Me ha llamado la atención el hecho de las condiciones que tienen, en comparación con España, basándome principalmente en el área de la ciencia. Se realizan numerosas contribuciones a la ciencia, se promueven distintos proyectos, con más facilidades que aquí. La libertad de la enseñanza y de la investigación está constitucionalmente garantizada, lo que supone que los centros tengan derechos de autogobierno. En resumen, me parece que está mejor organizada que en Espa$\tilde{n} a, y$ por lo que he leído en la prensa, los estudiantes reciben más becas y ayudas para salir adelante.

\section{Und Ana:}

Me ha llamado la atención, por lo que he leído, que la sociedad alemana es una sociedad en la que gran parte de la población son jóvenes. De entre las ideas de la juventud se puede observar que dan gran importancia a la familia y a la amistad así como a los estudios. Los jóvenes alemanes no están excesivamente interesados por la política pero les gusta comprometerse con la sociedad en la protección del medio ambiente o con actividades de voluntariado. Un gesto muy positivo es su lucha contra la xenofobia y el ultraderechismo.

Wie man sieht, entdecken die Lerner durch das Internet und das kulturelle Eintauchen in eine neue Welt Themen, die in keinem Lehrbuch auf der ersten Seite stehen. Das Forum dagegen baut eine Brükke zwischen dem ersten Kontakt im Klassenraum mit der Sprache und einem realen Kontext, wo sie auf die Kultur, Geschichte und Geographie eines Landes treffen, das sie mit ihrem eigenen in Vergleich setzen. Das Forum dient auf konstruktive Weise dazu, dass die Lerner auf eigene Weise eine neue Welt entdecken, das Internet als Informationsquelle und das Forum zum Austausch ihrer Eindrücke nutzen.

Allgemein festzustellen ist, dass das Forum ein idealer Ort für das Präsentieren von schriftlichen Ergebnissen ist: Die Kommentare und Beiträge werden im Verlauf des Kurses archiviert, die Lerner haben Zugang auch ausserhalb des Unterrichts, es können Themen und Aspekte angeboten werden, für die im Präsenzunterricht keine Zeit bleibt, und die Posts können auch Links zu Audio- und Videodokumenten beinhalten. Vor allem aber sei die Mitteilungsrichtung herausgestellt: alle können alles lesen, jeder kann schreiben, wo und wann er will.

\section{Mögliche Aufgabenstellungen für das Forum}

Die folgenden 10 Vorschläge stellen eine kleine Auswahl an möglichen Aufgabentypen dar. Sie sind als Ausgangspunkt gedacht, um mit dem Forum zu experimentieren, andere Varianten auszuprobieren oder einfach direkt loszulegen:

\section{Suchfragen}

- Der Kursleiter schreibt im Forum Fragen über wichtige Personen, die Geographie (Berge, Städte, Regionen, Sehenswürdigkeiten, Kuriositäten) oder über das aktuelle Geschehen. Es werden vor allem die W-Fragen aufgewärmt und die Lerner müssen recherchieren und Antworten suchen. 
Die Attrakkivität des e-Forums liegt darin, dass ein virtueller Ort die Teilnehmer über die Grenzen eines Klassenraumes, einer Stadt, eines Landes verbindet und zum sozialen Treffpunkt fiir den Austausch an Informationen und Emotionen wird.

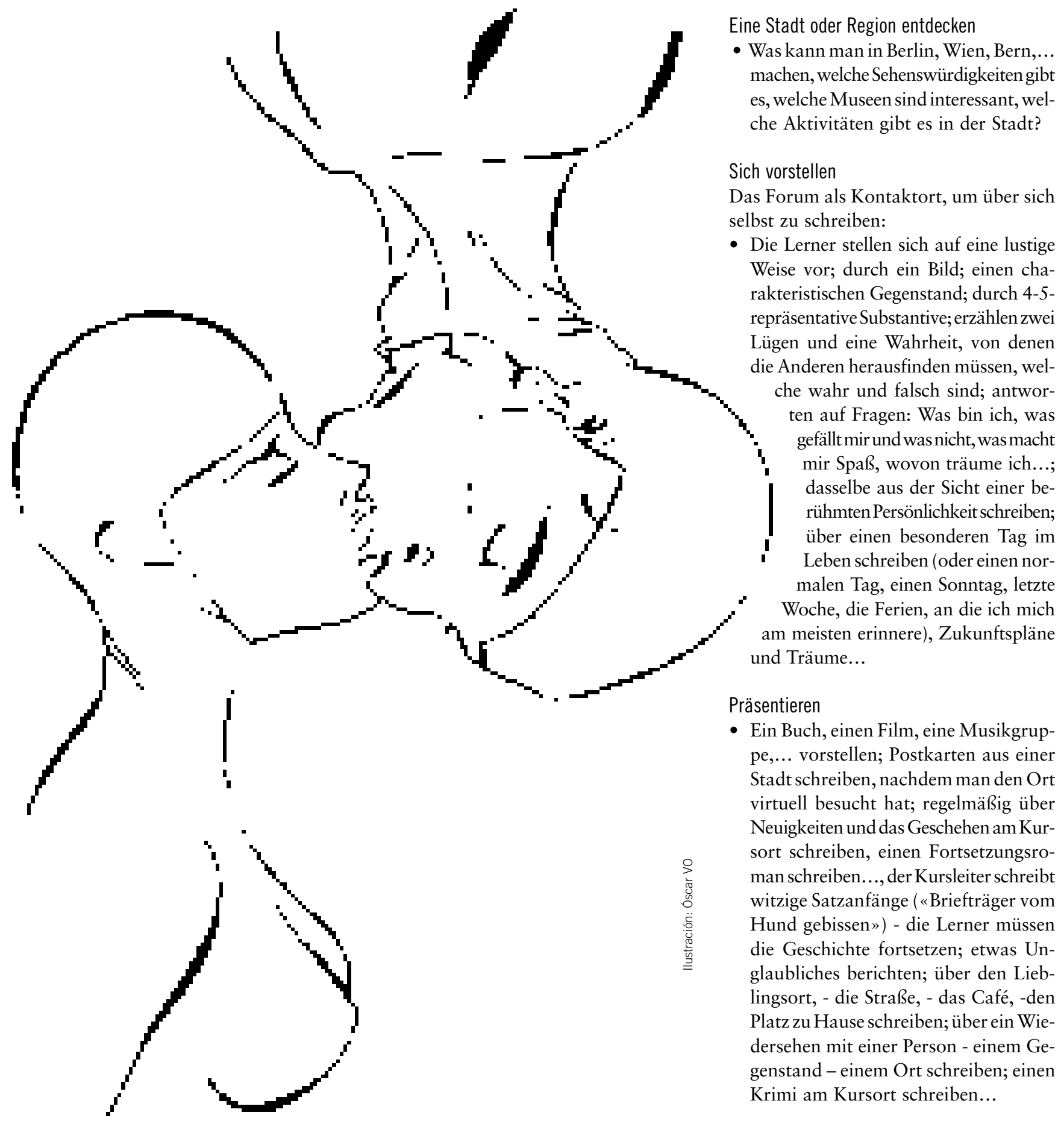


Sich in Andere hineinversetzen

- Bilder wie die von Edward Hopper benutzen und sich in die Person, an den Ort, in die Stimmung hineinversetzen, die Gedanken, Geschichten und Erinnerungen dieser Figuren beschreiben...,

- Aus der Sicht einer Filmfigur schreiben, eine charakteristische Situation beschreiben, die Anderen müssen raten, wer das ist.

\section{Das Forum als Lerntagebuch}

Das Forum kann auch ein Ort der regelmäßigen Reflexion über Lernfortschritte und -probleme sein. Es geht im Wesentlichen darum, Erfahrungen mitzuteilen, Fragen stellen, sich erkundigen, Gefundenes bekannt geben. Die Lerner können zum Beispiel (jede Woche) eine Zusammenfassung der letzten Lektion, eines Projekts oder einer Lektüre schreiben udn dabei besonders auf Aspekte eingehen wie: Was habe ich gelernt, was hat mir Schwierigkeiten bereitet, was möchte ich vertiefen...?

Eine mögliche Strukturierung könnte so aussehen: das Thema, die Situation, das Problem und den Kontext in Stichworten beschreiben, die Erfahrung zusammenfassen und einen Rückschluss für das weitere Vorgehen ziehen. Dieser metakognitive Prozess gehört u.a. zu den wesentlichen Grundzügen des autonomen Lernens und durch das Forum gewöhnen sich möglicherweise die Lerner daran, nicht nur über das eigene Lernen zu reflektieren, sondern ihre Lernerfahrung auch an Andere weiterzugeben, sie mit den Berichten der Anderen zu verlgeichen und so den Lernprozess sichtbar zu machen. Regelmäßige Beiträge dieser Art können auch einen Teil der formativen Evaluation bilden und in die Endbenotung einbezogen werden.

Eindrücke und Meinungen zu einem aktuellen Thema austauschen (Aufwärumübung für Meinungsäußerungen)

Nicht immer ist es leicht für die Lerner über die gestellen Themen im Unterricht spontan zu debattieren, und das nicht allein wegen sprachlicher Schwierigkeiten sondern, weil Kenntnisse über das Thema und Argumente fehlen. Wenn das Forum für Debatten genutzt wird, haben Lerner mehr Zeit sich über Positionen Gedanken zu machen und vor allem das The- ma vorher zu recherchieren: Im Forum können allgemeine Meinungsfragen gestellt werden wie: Was hältst du von..., was denkst du über...,

Als Grundlage kann ein Artikel gelesen werden, die Lerner sollen die positiven und negativen Aspekte herausstellen und darüber eine Diskussion eröffnen:

a) Jeder Teilnehmer schreibt dazu seine Meinung.

b) Kommentiert die Argumente der Anderen.

c) Schließt sich einer Meinung an oder verteidigt seine eigene Position

- Variante: drei dafür und dagegen: Die Lerner lesen in Dreiergruppen einen Artikel und schreiben einen Beitrag, indem sie: a) Argumente dafür und dagegen sammeln, b) eigene Positionen darstellen, c) die wichtigsten Fragen, die der Artikel aufwirft, herausstellen.

Relevante Webseiten zu einem Thema benutzen

Die Lerner besuchen eine Auswahl an relevanten Webseiten (eventuell in Paaren unterschiedliche Webseiten, Zeitungen oder andere Medien), geben den Inhalt wider und schreiben dazu ihre Meinung. Es kann noch eine Liste mit wichtigem Wortschatz dazu erstellt werden.

\section{Etwas kompliziertere Vorgehensweisen}

Jede Woche wird ein Moderator gewählt, der eine Frage im Forum schreibt, er ist in dieser Woche für das Moderieren zuständig (kommentiert die Beiträge, fügt Zusatzinformationen hinzu...), am Ende der Woche wird im Unterricht das Thema zusammengefasst und das nächste Thema und der Moderator gewählt.

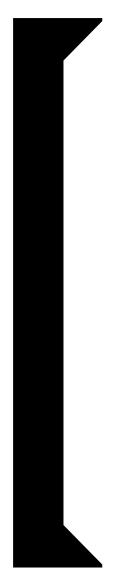

Offene Foren zum Deutschlernen im Internet Es gibt eine Reihe an Foren, die speziell an Deutschlerner gerichtet sind, den Austausch an Meinungen und die Verwendung des Deutschen weltweit fördern möchten.

Einige Beispiele sind: Die Deutsche Welle http://www.dw-world.de/. Das Kaleidoskop http://www.kaleidos.de/alltag/deindex.htm mit den Alltagstelegrammen über Feste, Eindrücke, Leute, Meinungen, etc.

Jetzt Deutsch lernen http://www.goethe.de/z/jetzt/, thematische Foren mit Aufgabenstellungen und Videos. Das Forum im Deutschlern Net http://www.deutschlern.net/

\section{Tipps zum Vorgehen}

Nicht alle Lerner sind mit einem Forum vertraut, deshalb ist eine erste Einführung im Unterricht notwendig. Ein Forum schläft auch nach kurzer Zeit ein, wenn der Kursleiter nicht motivierend mitwirkt, für die Lerner interessante Fragen schreibt, kommentiert und neue Gesichtspunte einbringt, die die Diskussionen animieren. Die Teilnahme am Forum ist produktiver, wenn die Gruppe zahlreich ist oder sogar gruppenübergreifend in einer bestimmten Niveaustufe diskutiert wird, da ein Forum mit drei Leuten schnell eintönig wird. Die Teilnahme hängt auch davon ab, ob ein Forum auf freiwilliger Basis angeboten wird oder Teil der Evaluation ist. Für die Endbenotung kann zum Beispiel eine bestimmte Anzahl an Beiträgen pro Monat verlangt und auf diese Weise die Produktivität forciert werden.

Sicherlich können die hier vorgestellten Aktivitäten ebenso ohne Forum während
Die Teilnahme am Forum ist produktiver, wenn die Gruppe zahlreich

ist oder sogar gruppenübergreifend in einer bestimmten Niveaustu-

fe diskutiert wird,da ein Forummit drei Leuten schnelle eintönig wird.

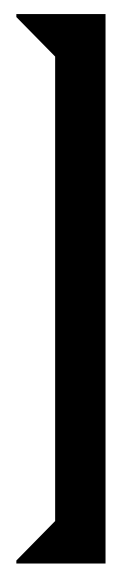


Wer es schafft ein Forum erfolgreich aufrechtzuerhallen, wird begeistertfeststellen, dassesaucheinenPlatzfürHumor,Fragen und Kommentare bietet, die häufiig besser auf schriftliche als auf mündliche Weise ausgedrückt werden.

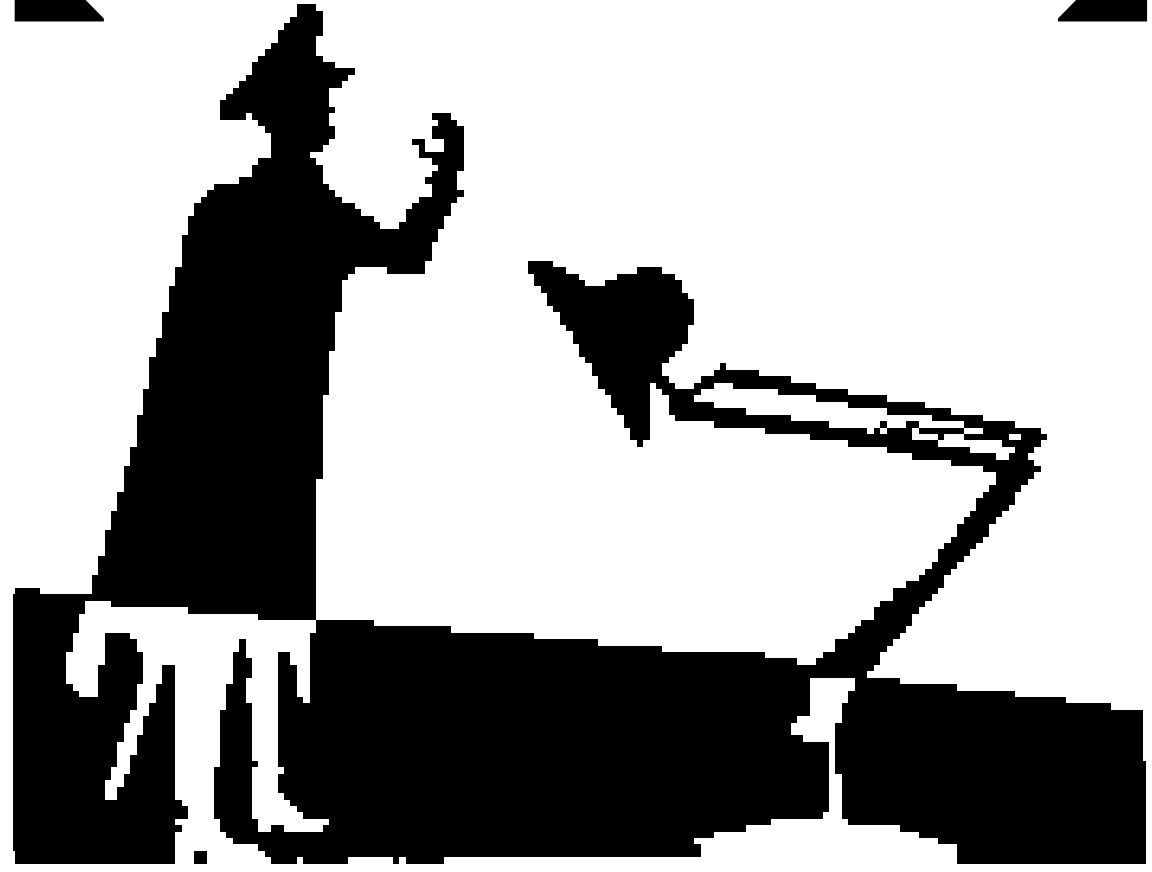

des Unterrichts angeboten werden, jedoch bietet die Arbeit mit dem Forum einige Vorteile, die den Präsenzunterricht bereichern können: die Zeit für den Kontakt mit der Sprache wird erweitert, Lerner mit zeitlichen Problemen und anderen Verpflichtungen finden trotz Abwesenheit Gelegenheit teilzunehmen, und im Falle einer kontinuierlichen Evaluation hat der Kursleiter ein weiteres Instrument zur Bewertung von Teilnahme und Lernfortschritten. Bei online oder blended-learning Kursen ist das Forum auf jeden Fall eine unerlässliche Komponente für die Kommunikation und den
Austausch, um sich auch über die Distanz hinweg kennenzulernen und mitzuarbeiten.

Wer es schafft ein Forum erfolgreich aufrechtzuerhalten, wird begeistert feststellen, dass es auch einen Platz für $\mathrm{Hu}-$ mor, Fragen und Kommentare bietet, die häufig besser auf schriftliche als auf mündliche Weise ausgedrückt werden. Auf jeden Fall ermöglicht die schriftliche Form mehr Zeit zum Ausformulieren, Korrigieren, sich Gedanken machen als das mündlich im Unterricht der Fall ist. Zusammenfassend überbrückt das Forum mögliche Nachteile des Klassenunterrichts, wie sie besonders bei sehr großen Gruppen in Universitätskursen auftreten können und den Kursleiter vor Probleme stellen, wenn es darum geht, Ergebnisse zu präsentieren. Während es im Unterricht dafür häufig an Zeit und nötiger Aufmerksamkeit mangelt, können im Forum alle zugleich schreiben und lesen, was Andere geschrieben haben.

Das Forum, wie auch die Verwendung von Chats und e-Mails, bietet Gelegenheit für kommunikative Aspekte des Spracherwerbs, es ist eine konstruktive Aktivität, fördert die Kolaboration und den Aufbau an Wissen, an dem die Teilnehmer teilhaben. Am Anfang gibt es nur eine leere Seite, die sich allmählich mit Beiträgen füllt, die über den Kursverlauf hinweg aufbewahrt werden. Sprachlernen wird vor allem zu einer sozialen und kommuniaktiven Handlung, die im Forum sichtbar gemacht wird. Alle können teilnehmen, von einander lernen, jeder kann etwas beitragen. Wenn Lernen als ein aktiver Prozess betrachtet wird, so bietet das Forum dafür ein brauchbares Werkzeug, indem es die Lerner zum Forschen, Lesen, Entdecken, Antworten suchen, Fragen und Kommunizieren animiert.

Die Arbeit mit einem Forum verdeutlicht meiner Meinung nach, was man als Lehrer idealerweise von seinen Schülern erwartet: eine aktive Haltung zum Lernen, die von jedem selbst abhängt, dass jeder etwas zum Kursinhalt beisteuern kann, wobei der Lehrer eine Stütze ist, aber für keinen den produktiven Teil übernehmen kann.

Nicht zuletzt tragen wir durch die Verwendung elektronischer Hilfsmittel zu der sogenannten neuen Lernkultur bei, indem Medien des technologischen Zeitalters für den Spracherwerb - dabei heben wir vor allem die sozialen und kulturellen Aspekte hervor - nutzbar gemacht werden

\section{Weiterführende Bibliographie}

Electronic Class Discussion: http://ftad.osu.edu/Publications/elecdisc/pages/advan.htm

LaGrandeur, Kevin, Using Electronic Discussion to Teach Literary Analysis, http://users.ox.ac.uk/ ctitext2/publish/comtxt/ct12/lagrand.html

Pérez Sánchez, Lourdes, "El foro virtual como espacio educativo: propuestas didácticas para su uso»,

Quaderns digitals $\mathrm{n}^{\circ} 40$, http://www.quadernsdigitals.net

Platten, Eva, «Die Bedeutung von Chats für das Fremdsprachenlernen», http://www.uni-giessen.de/lernwiki/chatfors/index.htm

Warschauer, Mark, «Comparing Face-to-Face and Electronic Discussion in the Second Language Classroom»,

http://www.gse.uci.edu/person/markw/comparing.html

- Beispiel für die Verwendung eines Forums als Lesetagebuch: http://www.online-lesetagebuch.de/lesetagebuch/forum_fragen.html 